\title{
Effectiveness of Allergic Rhinitis Management Related to WHO Guideline on Allergic Rhinitis and Its Impact on Asthma (ARIA)
}

\author{
Atika Aziza, ${ }^{1}$ Arif Dermawan, ${ }^{2}$ Vycke Yunivita Kusumah Dewi ${ }^{3}$ \\ ${ }^{1}$ Faculty of Medicine Universitas Padjadjaran, ${ }^{2}$ Department of Physical Medicine and \\ Rehabilitation Faculty of Medicine Universitas Padjadjaran/Dr. Hasan Sadikin General Hospital \\ Bandung, ${ }^{3}$ Department of Child Health Faculty of Medicine, Universitas Padjadjaran/Dr. Hasan \\ Sadikin General Hospital Bandung
}

\begin{abstract}
Background: The standard procedure of Allergic Rhinitis (AR) Management in Indonesia is based on Allergic Rhinitis and Its Impact on Asthma (ARIA) World Health Organization (WHO) 2008 guideline; however, it needs some adjustment to get an effective use locally in Indonesia. The data related to the problem however did not exist in Indonesia. The study aimed to evaluate the effectiveness of AR patient management based on the ARIA WHO guideline in the Department of Otorhinolaryngology-Head and Neck Surgery Dr. Hasan Sadikin General Hospital Bandung.

Methods: The study was conducted from September to October 2015 using quantitative descriptive design to observe the development of ARIA classification, total nasal symptom score (TNSS), and quality of life (QoL) during the first 6 months of therapy. The data were obtained from medical records of AR patients who visited the Rhinology-Allergy clinic Department of Otorhinolaryngology-Head and Neck Surgery Dr. Hasan Sadikin General Hospital within one year. Thirty three patients were included in the study using total sampling.

Results: There was significant improvement $(\mathrm{p}<0.001)$ in ARIA classification, TNSS, and QoL between the initiation of therapy, the third, and the sixth month. In contrary, there was no significant difference in ARIA classification ( $\mathrm{p}=0.109)$, TNSS $(\mathrm{p}=0.317)$, and QoL $(\mathrm{p}=1.000)$ between the third and the sixth month of therapy.

Conclusions: Allergic rhinitis patient management based on the 2008 ARIA WHO guideline is effective. [AM].2016;3(4):538-44]
\end{abstract}

Keywords: Allergic rhinitis, asthma, effectiveness, management

\section{Introduction}

Allergic Rhinitis (AR) is an inflammation of the nasal mucosa mediated by Immunoglobulin E (IgE) after exposure to allergen. The inflammatory reaction manifests as runny nose, nasal congestion, sneezing, and nasal itching. The clinical manifestation recurs after each exposure to the initiating allergen. ${ }^{1}$ Although there are not yet data on the national prevalence of AR in Indonesia, previous study conducted in 2010 at the Department of Otorhinolaryngology-Head and Neck Surgery Dr. Hasan Sadikin General Hospital Bandung showed the prevalence of AR is $24.5 \% .{ }^{2}$ Clinical manifestations of AR often cause impairment of quality of life (QoL). The impairment of QoL is caused by sleep disturbance and problems with social activities, school and work performance. ${ }^{1,3}$ This may lead to a decrease in productivity, and therefore impacts on the economy. ${ }^{3}$

According to the 2008 Allergic Rhinitis and its Impact on Asthma (ARIA) World Health Organization (WHO) guideline allergic rhinitis is classified based on disease severity and symptom duration. The classifications consist of mild intermittent AR (MI-AR), mild persistent AR (MP-AR), moderate/severe intermittent AR (MSI-AR), and moderate/ severe persistent AR (MSP-AR). These classifications determine the therapeutic plan of AR that includes allergen avoidance, patient education, pharmacotherapy and

Correspondence: Atika Aziza, Faculty of Medicine, Universitas Padjadjaran, Jalan Raya Bandung-Sumedang Km.21, Jatinangor, Sumedang, Indonesia, Phone: +62 85624985907 Email:atika.azii@gmail.com 
specific immunotherapy (SIT). According to the 2008 ARIA WHO recommendations, all four categories should undertake allergen avoidance and receive patient education and pharmacotherapy. Pharmacotherapy also differs from each classification and includes intranasal corticosteroid, $\mathrm{H} 1$ antihistamine, and leukotriene receptor antagonist (LTRA) among others. For persistent $\mathrm{AR}$, it is recommended that intranasal corticosteroid should be given as a first-line therapy. Specific immunotherapy is only recommended for MPAR, MSI-AR, and MSP-AR. ${ }^{1}$ Hence, only the three categories are recommended for the complete combined therapy. Even so, the management for AR patients may be adjusted accordingly if patients experience improvement or worsening in ARIA classification.

The management for ARIA in Indonesia has been performed according to the recommendations of the 2008 ARIA WHO. However, the 2008 ARIA WHO guideline is meant to be a guide to formulate an AR management guideline that is suitable to local environment and circumstances. ${ }^{1,4} \mathrm{Up}$ until now, there has been no data on the effectiveness of AR management based on the 2008 ARIA WHO guideline in Dr. Hasan Sadikin General Hospital or in any hospital in Indonesia. Therefore, an evaluation of the effectiveness of the 2008 ARIA WHO guideline in a local setting is needed. The evaluation of the 2008 ARIA WHO guideline recommendations effectiveness will be done based on guideline therapy goals that include improvements in ARIA classification, Total Nasal Symptom Score (TNSS), and patient QoL. Hence, the aim of this study is to evaluate the effectiveness of AR patient management according to the 2008 ARIA WHO guideline in the Rhinology-Allergy clinic of the Department of Otorhinolaryngology-Head and Neck Surgery Dr. Hasan Sadikin General Hospital.

\section{Methods}

This study was conducted in the RhinologyAllergy clinic of the Department of Otorhinolaryngology-Head and Neck Surgery Dr. Hasan Sadikin General Hospital and used a quantitative descriptive research design. The study was conducted from September to October 2015. The study samples were AR patients managed in the Rhinology-Allergy clinic that fulfilled the following inclusion criteria: patients classified as MP-AR, MSI$\mathrm{AR}$, and MSP-AR; patients having started SIT between March $1^{\text {st }} 2014$ and March $31^{\text {st }}$
2015; and patients having undertaken SIT for a minimum of 6 months. The samples which were not included were: patients starting SIT outside of the given time period, patients having undertaken SIT less than 6 months' time, and patients classified as MI-AR.

Study samples were taken according to total sampling method and this study included 33 AR patients who visited the Rhinology-Allergy clinic. The Subject data were taken from medical records, and permission regarding information disclosure was obtained through the Ethical Committee of Dr. Hasan Sadikin General Hospital and Faculty of Medicine Universitas Padjadjaran. The data were analyzed statistically to discover the variable frequency.

Research variables that were evaluated included the AR classification according to the 2008 ARIA WHO guideline, TNSS, and the QoL disturbance. Allergic rhinitis classifications consist of MSP-AR, MSI-AR, MP-AR, and MIAR. Total nasal symptom score was divided into very mild (a score of 0-2), mild (3-6), moderate (7-9), and severe (10-12) according to score presented in the medical record. Disturbance in QoL was recorded as those impaired and those not impaired. These variables were taken from the records of the patient's first visit (designated month 0), the third month of therapy (month 3) and the sixth month of therapy (month 6). The data were then analyzed to evaluate the frequency of each variable during each time frame to see differences between each time frame and also the significance of changes using Wilcoxon Signed-Rank test and McNemar's test. The result would be deemed significant if $p$ value was $<0.05$ and insignificant if $p$ value was $>0.05$.

The research variables were also included general characteristics such as age, gender and occupation, which would be presented as frequency. Comorbidities were also taken into account and were shown as frequency of those with comorbidities and those without along with the frequency of the types of comorbidities present.

\section{Results}

From 40 children, numbers of boy and girl in The subjects' general characteristics were mostly women $(69.7 \%)$ with the highest range of age in the 18-34 age group (42.4\%), followed by 35-49 age group (33.3\%) (Table $1)$. A majority of the subjects were school/ college students $(30.3 \%)$ and housewives 
Table 1 Subjects' General Characteristics

\begin{tabular}{lcc}
\hline \multirow{2}{*}{ Variable } & AR Patient & \\
\cline { 2 - 3 } & n & \% \\
\hline Gender & 23 & 69.7 \\
Female & 10 & 30.3 \\
Male & & \\
Age, range & 5 & 15.2 \\
$\leq 17$ & 14 & 42.4 \\
$18-34$ & 11 & 33.3 \\
$35-49$ & 3 & 9.1 \\
$50-64$ & 0 & 0 \\
$\geq 65$ & & \\
Occupation & 8 & 24.2 \\
Civil Servant & 5 & 15.2 \\
Private Employee & 11 & 33.3 \\
Student/College Student & 9 & 27.3 \\
Housewife & 0 & 0 \\
Unemployed & 0 & 0 \\
Others & & \\
Comorbidity & 20 & 60.6 \\
Present & 13 & 39.4 \\
Absent & & \\
\hline
\end{tabular}

Note; AR: Allergic rhinitis; n: Number of AR patient; \%: Percentage of AR patient

\section{(27.3\%).}

Comorbidities were also present in $60.6 \%$ of the subjects with the other 13 people without comorbidities. Rhinosinusitis was the most prevalent comorbidity in the study with a frequency of $50 \%$ (Table 2). The least prevalent comorbidity was conjunctivitis, otitis media, and atopic dermatitis with 5\% each.

At the beginning of the study the most common AR classification was based on the 2008 ARIA WHO guideline among the subjects was MSP-AR with $42.4 \%$ with no subjects classified as MI-AR (Table 3). All of the subjects had impaired QoL and most had moderate symptoms (60.6\%). By the 3rd

Table 2 Comorbidity Distribution

\begin{tabular}{lcc}
\hline \multirow{2}{*}{ Comorbidity } & \multicolumn{3}{c}{ AR Patient (n=20) } \\
\cline { 2 - 3 } & N & \% \\
\hline Rhinosinusitis & 10 & 50 \\
Nasal polyp & 5 & 25 \\
Asthma & 2 & 10 \\
Conjunctivitis & 1 & 5 \\
Atopic dermatitis & 1 & 5 \\
Otitis media & 1 & 5 \\
\hline
\end{tabular}

Note: AR: Allergic rhinitis; $n:$ Number of AR patient; \%: Percentage of AR patient 
Atika Aziza, Arif Dermawan, Vycke Yunivita Kusumah Dewi: Effectiveness of Allergic Rhinitis Management 541 Related to WHO Guideline on Allergic Rhinitis and Its Impact on Asthma (ARIA)

Table 3 ARIA Classification, TNSS and QoL Impairment Changes at Month 0, 3, and 6

\begin{tabular}{|c|c|c|c|c|c|c|c|c|c|}
\hline \multirow{3}{*}{ Variable } & \multicolumn{6}{|c|}{ AR Patient } & \multirow{3}{*}{$\begin{array}{c}\text { P value } \\
\text { (a)* }\end{array}$} & \multirow{3}{*}{$\begin{array}{c}\text { P value } \\
\text { (b) }\end{array}$} & \multirow{3}{*}{$\begin{array}{l}\text { P value } \\
\text { (c)\# }\end{array}$} \\
\hline & \multicolumn{2}{|c|}{ Month 0} & \multicolumn{2}{|c|}{ Month 3} & \multicolumn{2}{|c|}{ Month 6} & & & \\
\hline & $\mathrm{n}$ & $\%$ & $\mathbf{n}$ & $\%$ & $\mathbf{n}$ & $\%$ & & & \\
\hline \multicolumn{10}{|l|}{ ARIA Classification } \\
\hline MSP-AR & 22 & 66.7 & 2 & 6.1 & 0 & 0 & & & \\
\hline MSI-AR & 6 & 18.2 & 0 & 0 & 0 & 0 & \multirow{3}{*}{$<0.001$} & \multirow{3}{*}{0.109} & \multirow{3}{*}{$<0.001$} \\
\hline MP-AR & 5 & 15.2 & 1 & 3 & 1 & 3 & & & \\
\hline MI-AR & 0 & 0 & 30 & 90.9 & 32 & 97 & & & \\
\hline \multicolumn{10}{|l|}{ TNSS } \\
\hline Severe & 9 & 27.3 & 0 & 0 & 0 & 0 & \multirow[t]{4}{*}{$<0.001$} & \multirow[t]{4}{*}{0.317} & \multirow[t]{4}{*}{$<0.001$} \\
\hline Moderate & 20 & 60.6 & 0 & 0 & 0 & 0 & & & \\
\hline Mild & 4 & 12.1 & 1 & 3 & 3 & 9.1 & & & \\
\hline Very mild & 0 & 0 & 32 & 97 & 30 & 90.9 & & & \\
\hline \multicolumn{10}{|l|}{ QoL Impairment } \\
\hline Impaired & 33 & 100 & 3 & 9.1 & 3 & 9.1 & \multirow[t]{2}{*}{$<0.001$} & \multirow[t]{2}{*}{1.000} & \multirow[t]{2}{*}{$<0.001$} \\
\hline Not Impaired & 0 & 0 & 30 & 90.9 & 30 & 90.9 & & & \\
\hline
\end{tabular}

Note: AR: Allergic rhinitis; ARIA: Allergic Rhinitis and its Impact on Asthma; MSP: Moderate severe persistent; MSI: Moderate severe intermittent; MP: Mild persistent; MI: Mild intermittent; $n: N u m b e r$ of AR patient; \%: Percentage of AR patient, ${ }^{*} \mathrm{P}$ value (a) is the differences in variables between Month 0 and $3,{ }^{*} \mathrm{P}$ value (b) is the differences in variables between Month 3 and 6, \#P value (c) is the differences in variables between Month 0 and 6

month of therapy, the frequency of patients classified as MSP-AR was reduced to $6.1 \%$ with the highest frequency belonging to the MI-AR classification $(90.9 \%)$. The number of patients showing severe and moderate symptoms was decreased to zero and those with impaired QoL decreased to $9.1 \%$. More than a half of the subjects had no symptoms (69.7\%) and no QoL impairment (90.9\%). After 6 months of therapy, no subjects were classified as moderate/severe and only 3\% were MP-AR while the rest was MI-AR (97\%). Patients who did not present with QoL impairment were $90.9 \%$.

The result of statistical analysis using the Wilcoxon Signed-Rank test demonstrated

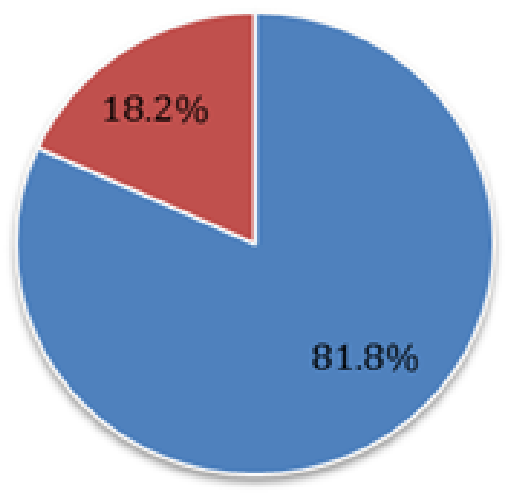

- H1 Antihistamine

- H1 Antihistamine

+ Intranasal

Corticosteroid

Figure Profile of Drug Use

Althea Medical Journal. 2016;3(4) 
that the ARIA classification and TNSS changes between start of therapy and the third month is significant with $\mathrm{p}$ value $<0.001$ (Table 3 ). This was also true of statistical analysis of the changes in QoL between the start of therapy with the third month of therapy using McNemar's test with $\mathrm{p}$ value $<0.001$. The difference between the start of therapy and the sixth month showed similar results to $p$ value $<0.001$ for each variable. Even so, it was found that the difference in ARIA classification, TNSS, and QoL between the third month and sixth month is not significant.

The distribution of drug combinations used in pharmacotherapy of subjects at the start of therapy showed that the highest frequency of drug used is $\mathrm{H} 1$ antihistamine (Figure). The other $18.2 \%$ was the combination of $\mathrm{H} 1$ antihistamine and intranasal corticosteroid.

\section{Discussions}

This study reveals that the distribution of gender, age, occupation, and presence of comorbidity of the study subjects is similar to the previous study in 2014 of AR patients visiting Department of OtorhinolaryngologyHead and Neck Surgery Dr. Hasan Sadikin General Hospital. ${ }^{5}$ Study subjects consist mostly of women $(69.7 \%)$. This high prevalence in women is thought to be due to hormonal differences between genders, in which oestrogen is known to be pro-inflammatory and thus predispose to atopy. ${ }^{6}$ The occupation distribution is also similar, with the highest being school/college students (30.3\%) and the second highest being housewives $(27.3 \%)$. It is known that AR affects school age children and thus causes learning disturbance. ${ }^{1}$ The study subjects are mostly between 18-34 years of age $(42.4 \%)$ and the trend declines with increase in age. The previous study showed a decrease in atopy with aging, it is suggested that a decrease in allergen-specific IgE concentration is the cause of this phenomenon. ${ }^{7}$

Most of the study subjects present with comorbidity $(60.6 \%)$ with rhinosinusitis as the highest frequency (55.6\%). This is in line with the previous study that rhinosinusitis is the most prevalent comorbidity in $\mathrm{AR}$ patients.2The presence of comorbidity may affect treatment outcome as the most of the comorbidity have the same pathophysiology as AR. The presence of comorbidity in study subjects may affect changes in ARIA classification, TNSS, or QoL disturbances in this study.
There is a significant change in distribution of ARIA classification between the first month of therapy and third month, and also with the sixth month of therapy. The frequency of MSP-AR and MSI-AR decreases between the start of therapy and the sixth month in which the frequency of MSP-AR is as high as $66.7 \%$ and MSI-AR as high as $18.2 \%$ at the start and only $6.1 \%$ and none respectively in the sixth month (Table 3). These findings indicate that patients given therapy according to ARIA WHO 2008 guideline experience an improvement in ARIA classification. These improvements are significant with $\mathrm{p}$ value $<0.001$. A previous study in Spain $^{8}$ support these findings that AR patients with moderate-severe classification experience a significant decrease in disease severity to mild after undergoing 4 weeks of pharmacotherapy with second generation $\mathrm{H} 1$ antihistamine.

The TNSS and QoL also show significant improvement $(p<0.001)$ before and after 6 months of therapy. At the start of therapy a majority of the patients experience severe symptoms while at the sixth month most of patients' symptoms improve to very mild (Table 3). Quality of life impairment distribution also shows significant change $(p<0.001)$ and by the sixth month of therapy $90.9 \%$ of patients experience no impairment. These findings are in line with a previous study in which ARIA recommended pharmacotherapy significantly improve patients' TNSS, QoL score, and disease severity after 4 weeks' time. A different study also shows that SIT is effective in reducing symptoms and medication usage in $A R$ patients. $^{10}$

The distribution change between the start of therapy and the third month with the change between the third and sixth month shows disparity. The distribution change of ARIA classification, TNSS, and QoL between the start and third month of therapy is larger than the change between the third and sixth month. These differences are only significant between the start of therapy and third month but not between the third and sixth month. This occurrence may be explained by the discoveries in a previous study that the largest improvement of QoL score occur in the first week of therapy and the largest improvement of TNSS occur after two weeks of therapy using second generation $\mathrm{H} 1$ antihistamine. ${ }^{11} \mathrm{~A}$ different study shows that as much as $52.6 \%$ of AR patients with moderate-severe symptoms experience improvement after 4 weeks of pharmacotherapy $(\mathrm{p}<0.0001) .{ }^{9}$

On another note, the TNSS of 2 subjects 
(6.1\%) showed worsening between the third and sixth month of therapy, changing from very mild to mild. Also, there is a persistence of QoL impairment in 3 subjects $(9.1 \%)$ in the same time frame. This TNSS worsening and persistence of impairment may be due to the presence of comorbidity, which is prevalent in this study. Comorbidity is known to affect therapy outcome, TNSS and QoL of AR patients. ${ }^{12}$ Therefore, the worsening of subjects' TNSS and persistence of QoL impairment is thought to be due to the presence of comorbidity, which affects the TNSS and QoL of subjects.

A majority of the study subjects receive cetirizine, a second generation $\mathrm{H} 1$ antihistamine, as opposed to intranasal corticosteroid such as fluticasone propionate which is the first-line medication for MSPAR patients according to ARIA WHO 2008 guideline. ${ }^{1}$ It is known that intranasal corticosteroid is more effective in reducing AR symptoms than $\mathrm{H} 1$ antihistamine ${ }^{14}$, and yet patients are instead primarily given $\mathrm{H} 1$ antihistamine and is only given intranasal corticosteroid in combination with $\mathrm{H} 1$ antihistamine. This discrepancy is due to the availability of medication in the clinic and hospital policy where currently only H1 antihistamine is readily available rather than intranasal corticosteroid.

During the course of this study there were several difficulties in obtaining data from the medical record. Some of this is caused by poor documentation due to unintelligible handwriting. Unsystematic medical record and storage also hindered data collection.

This is the first study to evaluate the effectiveness of AR management based on 2008 ARIA WHO guideline in Dr. Hasan Sadikin General Hospital. There are many studies about the effectiveness and efficacy of pharmacotherapy or SIT alone but none about the effectiveness of ARIA WHO 2008 guideline or the combined effectiveness of pharmacotherapy and SIT.

In summary, despite the discrepancy between practice and ARIA recommendations, AR patient management based on the 2008 ARIA WHO guideline proves to be effective. Even if local circumstance, which in this case limits the drug availability, hinder the application of ARIA recommendation, the guideline is suitable for the local setting in Dr. Hasan Sadikin General Hospital. Thus, the 2008 ARIA WHO guideline is appropriate for use in other healthcare facilities that resembles the setting of this study and as such may be used as guideline for management of local AR patients.

\section{References}

1. Bousquet J, Khaltaev N, Cruz AA, Denburg J, FokkensWJ, Togias A, et al. Allergic rhinitis and its impact on asthma (ARIA) 2008. Allergy. 2008;63(Suppl86):S8-160.

2. Achmad Sodikin, Teti Madiadipoera. Karakteristik penderita rhinitis alergi sesuai guideline allergic rhinitis and its impact on asthma (ARIA) di bagian THTKL RS. Hasan Sadikin Bandung periode 1 Januari - 31 Desember 2009. Proceedings of The 6th Jakarta International Functional Endoscopic Sinus Surgery Course and Workshop; 2010 Mar 4-7; Jakarta. Jakarta: Perhimpunan Dokter Ahli THT-KL; 2010.

3. Katelaris $\mathrm{CH}$, Lai CK, Rhee CS, Lee SH, Yun WD, Lim-Varona L, et al. Nasal allergies in the Asian-Pacific population: results from the allergies in Asia-Pacific survey. Am J Rhinol Allergy. 2011;25(Suppl1):S3-15.

4. BrożekJL, Bousquet J, Baena-Cagnani CE, Bonini S, CanonicaGW, Casale TB, et al. Allergic rhinitis and its impact on asthma (ARIA) guidelines: 2010 revision. J Allergy ClinImmunol. 2010;126(3):466-76.

5. Raissa Mentari Moeis, Melati Sudiro, RB. Soeherman Herdiningrat. Allergic rhinitis patient characteristics in Dr. Hasan Sadikin General Hospital Bandung in Indonesia. AMJ. 2014;1(2):75-80.

6. Osman M, Hansell AL, Simpson CR, Hollowell J, Helms PJ. Gender-specific presentations for asthma, allergic rhinitis and eczema in primary care. Prim Care Respir J. 2007;16(1):28-35.

7. Scichilone N, Callari A, Augugliaro G, Marchese M, Togias A, Bellia V. The impact of age on prevalence of positive skin prick tests and specific IgE tests. Respir Med. 2011;105(5):651-8.

8. Valero A, Izquierdo I, Giralt J, Bartra J, del Cuvillo A, Mullol J. Rupatadine improves nasal symptoms, quality of life (ESPRINT-15) and severity in a subanalysis of a cohort of Spanish allergic rhinitis patients. J Investig Allergol Clin Immunol. 2011;21(3):229-35.

9. Mullol J, Bartra J, del Cuvillo A, Izquierdo I, Muñoz-Cano R, Valero A. Specialist-based treatment reduces the severity of allergic rhinitis. Clin Exp Allergy. 2013;43(7):7239.

10. Dretzke J, Meadows A, Novielli N, Huissoon A, Fry-Smith A, Meads C. Subcutaneous 
and sublingual immunotherapy for seasonal allergic rhinitis: a systematic review and indirect comparison. J Allergy ClinImmunol. 2013;131(5):1361-6.

11. Holmberg K, Tonnel AB, Dreyfus I, Olsson P, Cougnard J, Mesbah K, et al. Desloratadine relieves nasal congestion and improves quality-of-life in persistent allergic rhinitis. Allergy. 2009;64(11):1663-70.

12. Feng CH, Miller MD, Simon RA. The united allergic airway: Connections between allergic rhinitis, asthma, and chronic sinusitis. Am J Rhinol. 2012;26(3):187-90.
13. Devillier P, Dreyfus JF, Demoly P, Calderon MA. A meta-analysis of sublingual allergen immunotherapy and pharmacotherapy in pollen-induced seasonal allergic rhino conjunctivitis. BMC Med. 2014;12(1):112.

14. Benninger M, Farrar JR, Blaiss M, Chipps B, Ferguson B, Krouse J, et al. Evaluating approved medications to treat allergic rhinitis in the United States: an evidencebased review of efficacy for nasal symptoms by class. Ann Allergy Asthma Immunol. 2010;104(1):13-29 